ARTICLE

\title{
Converting microwave and telecom photons with a silicon photonic nanomechanical interface
}

\author{
G. Arnold ${ }^{1,3}$, M. Wulf ${ }^{1,3}$, S. Barzanjeh ${ }^{1,2}$, E. S. Redchenko ${ }^{1}$, A. Rueda (1) ${ }^{1}$, W. J. Hease ${ }^{1}$, F. Hassani ${ }^{1} \&$ \\ J. M. Fink (D) ${ }^{1 凶}$
}

Practical quantum networks require low-loss and noise-resilient optical interconnects as well as non-Gaussian resources for entanglement distillation and distributed quantum computation. The latter could be provided by superconducting circuits but existing solutions to interface the microwave and optical domains lack either scalability or efficiency, and in most cases the conversion noise is not known. In this work we utilize the unique opportunities of silicon photonics, cavity optomechanics and superconducting circuits to demonstrate a fully integrated, coherent transducer interfacing the microwave $\mathrm{X}$ and the telecom $\mathrm{S}$ bands with a total (internal) bidirectional transduction efficiency of $1.2 \%$ (135\%) at millikelvin temperatures. The coupling relies solely on the radiation pressure interaction mediated by the femtometer-scale motion of two silicon nanobeams reaching a $V_{\pi}$ as low as $16 \mu \mathrm{V}$ for subnanowatt pump powers. Without the associated optomechanical gain, we achieve a total (internal) pure conversion efficiency of up to $0.019 \%(1.6 \%)$, relevant for future noise-free operation on this qubit-compatible platform.

\footnotetext{
${ }^{1}$ Institute of Science and Technology Austria, Am Campus 1, 3400 Klosterneuburg, Austria. ${ }^{2}$ Present address: Institute for Quantum Science and Technology (IQST), University of Calgary, Calgary, AB, Canada. ${ }^{3}$ These authors contributed equally: G. Arnold, M. Wulf. ${ }^{凶}$ email: jfink@ist.ac.at
} 
arge scale quantum networks will facilitate the next level in quantum information technology, such as the internet did for classical communication, enabling, e.g., secure communication and distributed quantum computation ${ }^{1}$. Some of the most promising platforms to process quantum information locally, such as superconducting circuits ${ }^{2}$, spins in solids ${ }^{3}$, and quantum dots $^{4}$, operate naturally in the gigahertz frequency range, but the long-distance transmission of gigahertz radiation is relatively lossy and not resilient to ambient noise. This limits the length of supercooled microwave waveguides in a realistic scenario to tens of meters ${ }^{5}$. In contrast, the transport of quantum information over distances of about $100 \mathrm{~km}$ is nowadays routinely achieved by sending optical photons at telecom frequency through optical fibers.

There is a variety of platforms, which in principle have shown to be able to merge the advantages of both worlds ranging from mechanical, piezoelectric, electro-optic, magneto-optic, rareearth, and Rydberg atom implementations ${ }^{6,7}$. So far, the optomechanical approach ${ }^{8}$ has been proven to be most efficient, reaching a record high photon conversion efficiency of up to $47 \%$ with an added noise photon number of only $38^{9}$. But this composite device is based on a Fabry-Perot cavity that has to be hand assembled and utilizes a membrane mode that is restricted to relatively low mechanical frequencies. Using piezo-electricity, coherent conversion between microwave and optical frequencies has been shown uni- and bidirectional at room temperature ${ }^{10,11}$, and at low temperatures ${ }^{12,13}$ with integrated devices, so far with either low efficiency or unspecified conversion noise properties. The electro-optic platform has shown promising photon conversion efficiencies ${ }^{14}$, recently up to $2 \%$ at $2 \mathrm{~K}^{15}$, but generally requires very large pump powers in the milliwatt range ${ }^{16}$.

In this work we present a device that converts coherent signals between $10.5 \mathrm{GHz}$ and $198 \mathrm{THz}$ at millikelvin temperatures via the radiation pressure interaction. Due to the need of only picowatt range pump powers, both the heat load to the cryostat and local heating of the integrated device is minimized. The chipscale device is fabricated from CMOS compatible materials on a commercial silicon-on-insulator wafer over an area of $\sim 200 \mu \mathrm{m} \times$ $120 \mu \mathrm{m}$. It is compact, versatile and fully compatible with silicon photonics ${ }^{17}$ and superconducting qubits ${ }^{18}$. The unique electroopto-mechanical design is optimized for very strong field confinements and radiation pressure couplings, which enable internal efficiencies exceeding unity for ultra-low pump powers. We find that the conversion noise so far precludes a quantum limited operation and we present a comprehensive theoretical and experimental noise analysis to evaluate the potential for scalable and noise-free conversion in the future. Such a power-efficient, ultra-sensitive, and highly integrated hybrid interconnect might find applications ranging from quantum communication ${ }^{8}$ and RF receivers ${ }^{19}$ to magnetic resonance imaging ${ }^{20}$.

\section{Results}

Transducer theory. The transducer consists of one microwave resonator and one optical cavity, both parametrically coupled via the vacuum coupling rates $g_{0, j}$ with $j=e, o$ to the same mechanical oscillator as shown in Fig. la and b. The intrinsic decay rate of the optical (microwave) resonator is $\kappa_{\text {in,o }}\left(\kappa_{\text {in,e }}\right)$, while the optical (microwave) waveguide-resonator coupling is given by $\kappa_{\text {ex,o }}\left(\kappa_{\text {ex,e }}\right)$ resulting in a total damping rate of $\kappa_{\mathrm{j}}=\kappa_{\mathrm{in}, \mathrm{j}}+\kappa_{\mathrm{ex}, \mathrm{j}}$ and coupling ratios $\eta_{\mathrm{j}}=\kappa_{\mathrm{ex}, \mathrm{j}} / \kappa_{\mathrm{j}}$. The mechanical oscillator with intrinsic decoherence rate $\gamma_{\mathrm{m}}$ and frequency $\omega_{\mathrm{m}}$ is shared between the optical cavity and the microwave resonator and acts as a bidirectional coherent pathway to convert the photons between the two different frequencies ${ }^{8,21-23}$. In the interaction frame, the Hamiltonian describing the conversion process is (see Supplementary Note 1):

$$
\hat{H}_{\mathrm{int}}=\sum_{\mathrm{j}=\mathrm{e}, \mathrm{o}}\left(\hbar G_{\mathrm{j}}\left(\hat{a}_{\mathrm{j}}^{\dagger} \hat{b}+\hat{a}_{\mathrm{j}} \hat{b}^{\dagger}\right)+\hat{H}_{\mathrm{CR}, \mathrm{j}}\right),
$$

where $\hat{a}_{\mathrm{j}}$, $(\hat{b})$ with $j=e, o$ is the annihilator operator of the electromagnetic (mechanical) mode, and $\hat{H}_{\mathrm{CR}, \mathrm{j}}=\hbar G_{\mathrm{j}}\left(\hat{a}_{\mathrm{j}} \hat{b} e^{2 i \omega_{\mathrm{m}} t}+\right.$ h.c.) describes the counter-rotating terms which are responsible for the coherent amplification of the signal. $G_{\mathrm{j}}=\sqrt{n_{\mathrm{d}, \mathrm{j}}} g_{0, \mathrm{j}}$ is the parametrically enhanced electro- or optomechanical coupling rate where $n_{\mathrm{d}, \mathrm{j}}$ is the intracavity photon number due to the corresponding microwave and optical pump tones. For a red-detuned drive in the resolved-sideband regime $4 \omega_{\mathrm{m}}>\kappa_{\mathrm{j}}$ we neglect $\hat{H}_{\mathrm{CR}, \mathrm{j}}$ under the rotating-wave approximation and the Hamiltonian (1) represents a beam-splitter like interaction in which the mechanical resonator mediates noiseless photon conversion between microwave and optical modes. Note that near-unity photon conversion $\zeta_{\mathrm{RS}}=4 \eta_{\mathrm{e}} \eta_{\mathrm{o}} \mathcal{C}_{\mathrm{e}} \mathcal{C}_{\mathrm{o}} /\left(1+\mathcal{C}_{\mathrm{e}}+\mathcal{C}_{\mathrm{o}}\right)^{2}$ can be achieved in the limit of $\mathcal{C}_{\mathrm{e}}=\mathcal{C}_{\mathrm{o}} \gg 1$ with $\mathcal{C}_{\mathrm{j}}=4 G_{\mathrm{j}}^{2} /\left(\kappa_{\mathrm{j}} \gamma_{\mathrm{m}}\right)$ being the electro- or optomechanical cooperativity, as demonstrated between two optical ${ }^{24}$ and two microwave modes $^{25,26}$, respectively.

Transducer design. We realize conversion by connecting an optomechanical photonic crystal zipper cavity ${ }^{27}$ with two aluminum coated and mechanically compliant silicon nanostrings ${ }^{28}$ as shown in Fig. 1c. The mechanical coupling between these two components is carefully designed (see Supplementary Note 2), leading to a hybridization of their in-plane vibrational modes into symmetric and antisymmetric supermodes. In case of the antisymmetric mode that is used in this experiment, the strings and the photonic crystal beams vibrate $180^{\circ}$ out of phase as shown by the finite-element method simulation in Fig. 1d. The photonic crystal cavity features two resonances at telecom frequencies with similar optomechanical coupling strength. The simulated spatial distribution of the electric field component $E_{\mathrm{y}}(x, y)$ of the higher frequency mode with lower loss rate used in the experiment is shown in Fig. 1e. The lumped element microwave resonator consists of an ultra-low stray capacitance planar spiral coil inductor ${ }^{29}$ and two mechanically compliant capacitors with a vacuum gap of size of $\sim 70 \mathrm{~nm}$. This resonator is inductively coupled to a shorted coplanar waveguide, which is used to send and retrieve microwave signals from the device. The sample is fabricated using a robust multi-step recipe including electron beam lithography, silicon etching, aluminum thin-film deposition, and hydrofluoric vapor acid etching, as described in detail in ref. ${ }^{30}$.

Transducer characterization. Standard sample characterization (see Supplementary Notes 3 and 4) reveals an optical resonance frequency of $\omega_{\mathrm{o}} /(2 \pi)=198.081 \mathrm{THz}$ with total loss rate $\kappa_{\mathrm{o}} /(2 \pi)=$ $1.6 \mathrm{GHz}$ and waveguide coupling rate $\kappa_{\mathrm{ex}, \mathrm{o}} /(2 \pi)=0.18 \mathrm{GHz}$ leading to a coupling efficiency of $\eta_{\mathrm{o}}=0.11$. When the optical light is turned off, the microwave resonance frequency is $\omega_{\mathrm{e}} /(2 \pi)=$ $10.5 \mathrm{GHz}$ with coupling efficiency $\eta_{\mathrm{e}}=0.4$ and $\kappa_{\text {ex,e }} /(2 \pi)=$ $1.15 \mathrm{MHz}$. The mechanical resonator frequency has a value of $\omega_{\mathrm{m}} /(2 \pi)=11.843 \mathrm{MHz}$ with an intrinsic decoherence rate $\gamma_{\mathrm{m}} /(2 \pi)=15 \mathrm{~Hz}$ at a mode temperature of $150 \mathrm{mK}$. The achieved single-photon-phonon coupling rates are as high as $g_{0, \mathrm{e}} /(2 \pi)=$ $67 \mathrm{~Hz}$ and $g_{0, \mathrm{o}} /(2 \pi)=0.66 \mathrm{MHz}$.

Conversion measurements. To perform coherent photon conversion, red-detuned microwave and optical tones with powers $P_{\mathrm{e}(\mathrm{o})}$ are applied to the microwave and the optical resonator. These drive tones establish the linearized electro- and optomechanical interactions, which results in the conversion of a weak 
a

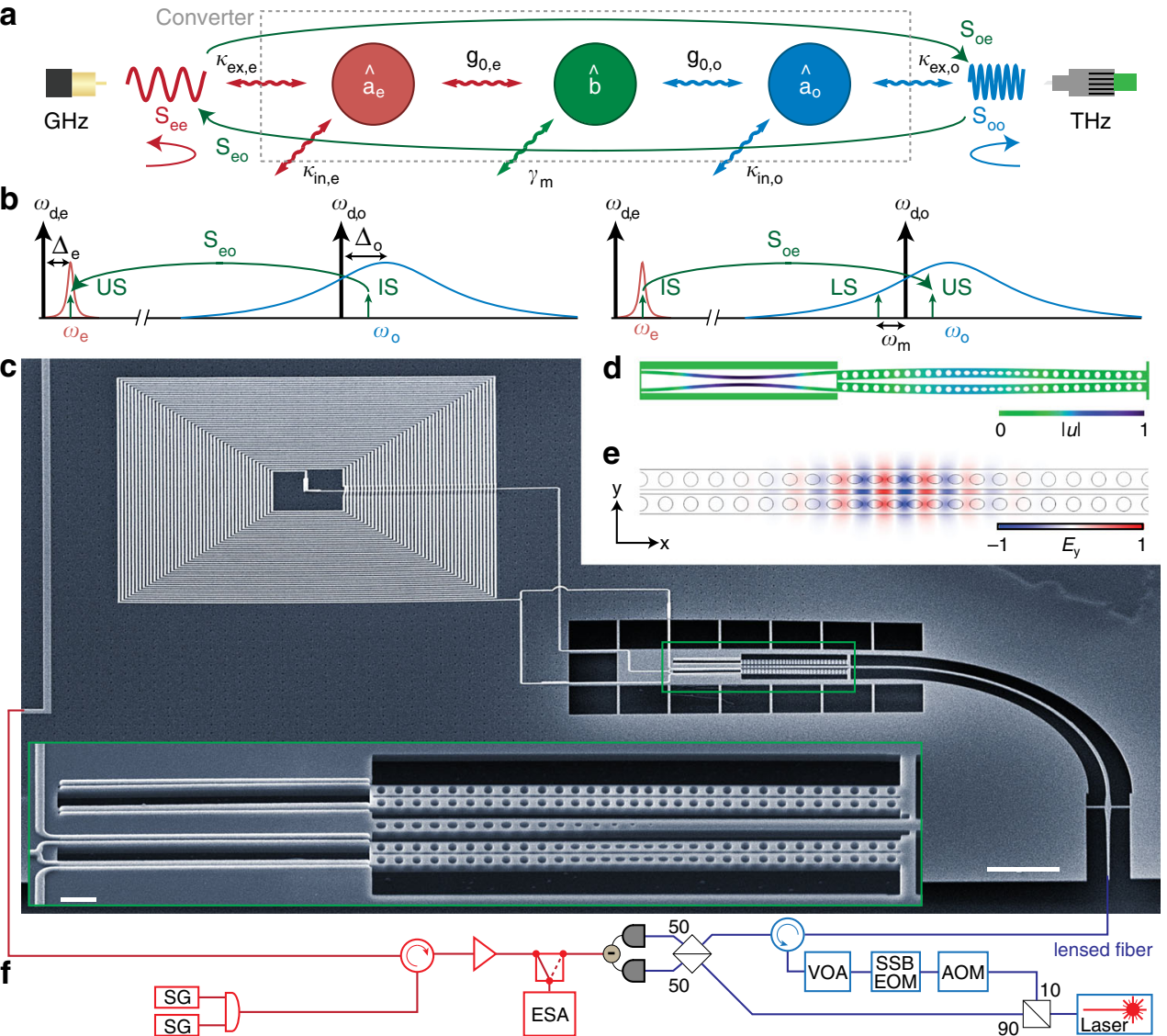

Fig. 1 Silicon photonic microwave-to-optics converter. a Diagram showing the microwave $\left(\hat{a}_{\mathrm{e}}\right)$, mechanical $(\hat{b})$, and optical $\left(\hat{a}_{\mathrm{o}}\right)$ mode, and the relevant coupling and loss rates of the device. Scattering parameters $S_{\mathrm{ij}}$ characterizing the transducer performance are indicated. $\mathbf{b}$ Schematic showing the frequencies of the coherent signals involved in the conversion process (green arrows). On the left an optical input signal (IS) is converted to an upper sideband (US) of the microwave pump signal, whereas in the microwave-to-optics conversion on the right also the lower sideband (LS) is created. The bidirectional transduction $\zeta$ is only evaluated between the upper sidebands at $\omega_{\mathrm{d}, \mathrm{j}}+\omega_{\mathrm{m}}$. c Scanning electron micrograph of the device showing the microwave lumped element resonator with an inductively coupled feed line, the photonic crystal cavity, and the optical coupling waveguide fabricated on a fully suspended $220 \mathrm{~nm}$ thick silicon-on-insulator device layer. White scale bar, $10 \mu \mathrm{m}$. The inset shows an enlarged view of the central part (green boxed area) comprising the mechanically compliant vacuum gap capacitors of size $\sim 70 \mathrm{~nm}$ and two optomechancial zipper cavities (top one unused) with a central tapered photonic crystal mirror coupled optical waveguide. White scale bar, $1 \mu \mathrm{m}$. d Finite-element method (FEM) simulation of the mechanical displacement $|u|$ of the utilized mechanical resonance. e FEM simulation of the electric in-plane-field $E_{y}(x, y)$ for the relevant optical mode. $\mathbf{f}$ Simplified experimental setup. The device is mounted on the mixing chamber plate of a cryogen-free dilution refrigerator at a temperature of $T_{\text {fridge }}=50 \mathrm{mK}$. A microwave switch selects between the incoming microwave and optical signal to be analyzed by the ESA. Optical heterodyning is used to detect the low power levels used in the experiment. SG microwave signal generator; ESA electronic spectrum analyzer; VOA variable optical attenuator; SSB EOM singlesideband electro-optic modulator; AOM acousto-optic modulator.

microwave (optical) signal tone to the optical (microwave) domain measured in our setup as shown in Fig. 1f. We experimentally characterize the transducer efficiency by measuring the normalized reflection $\left|S_{\mathrm{jj}}\right|^{2}(j=e, o)$ and the bidirectional transmission $\zeta:=\left|S_{\text {eo }}\right|\left|S_{\text {oe }}\right|$ coefficients as a function of signal detuning $\delta_{j}$. As shown in Fig. $2 \mathrm{a}$, for drive powers $P_{\mathrm{e}}=601 \mathrm{pW}$ and $P_{\mathrm{o}}=$ $625 \mathrm{pW}$ with drive frequencies $\omega_{\mathrm{d}, \mathrm{j}}$ and detunings $\Delta_{\mathrm{j}}=\omega_{\mathrm{j}}-\omega_{\mathrm{d}, \mathrm{j}}$ of $\Delta_{\mathrm{e}}=\omega_{\mathrm{m}}$ and $\Delta_{\mathrm{o}} /(2 \pi)=126 \mathrm{MHz}$ leading to intracavity photon numbers of $n_{\mathrm{d}, \mathrm{e}} \approx 9 \times 10^{5}$ and $n_{\mathrm{d}, \mathrm{o}} \approx 0.2$ with cooperativities $\mathcal{C}_{\mathrm{e}} \approx 0.57$ and $\mathcal{C}_{\mathrm{o}} \approx 0.9$, the measured total (waveguide to waveguide) photon transduction efficiency is $\approx 1.1 \%$ corresponding to $96.7 \%$ internal (resonator to resonator) photon transduction efficiency over the total bandwidth of $\Gamma_{\text {conv }} /(2 \pi) \approx$ $0.37 \mathrm{kHz}$. In the case of $\kappa_{\mathrm{o}}>4 \omega_{\mathrm{m}}$ and $\kappa_{\mathrm{e}}<4 \omega_{\mathrm{m}}$, the bandwidth is given by $\Gamma_{\text {conv }} \approx\left(\mathcal{C}_{\mathrm{e}}+1\right) \gamma_{\mathrm{m}}$ because the nonsideband resolved optomechanical cavity does not induce mechanical broadening. The signal tone adds $17\left(10^{-3}\right)$ photons to the microwave resonator (optical cavity).
Here we use a self-calibrated measurement scheme that is independent of the gain and loss of the measurement lines as described in ref. ${ }^{31}$ and we only take into account transduction between the upper two sidebands at $\omega_{\mathrm{d}, \mathrm{j}}+\omega_{\mathrm{m}}$ as shown in Fig. 1b. Neglecting the lower optical sideband that is generated due to the nonsideband resolved situation $\kappa_{\mathrm{o}} / 4 \omega_{\mathrm{m}} \approx 30$ reduces the reported mean bidirectional efficiencies by $\sqrt{2}$ compared to the actually achieved total transduction efficiency between microwave and optical fields. The observed reflection peaks indicate that both resonators are undercoupled, equivalent to an impedance mismatch for incoming signal light. All scattering parameters are obtained from measured coherent tones whose linewidths are given by the chosen resolution bandwidth and the stability of the heterodyne setup. While this does not explicitly show long term phase stability of the conversion we find that these results are in excellent agreement with our coherent conversion theory model (solid lines) with $\gamma_{\mathrm{m}}$ as the only free fit parameter. 
a
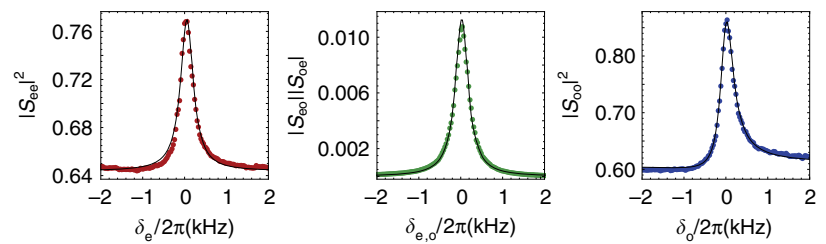

b

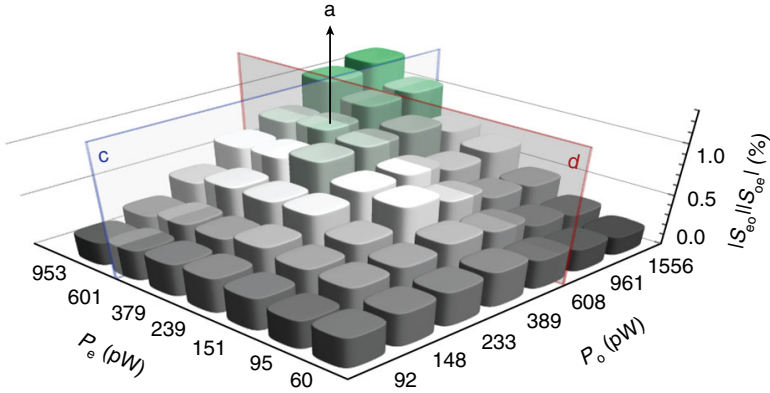

C

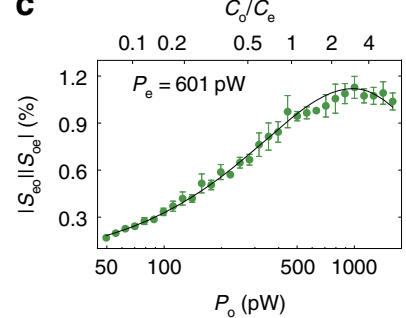

d

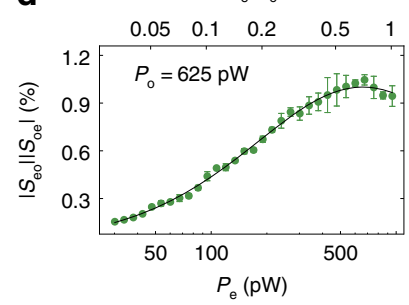

e

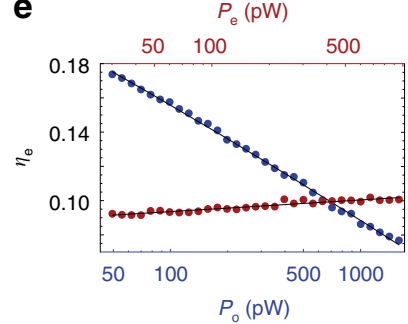

f

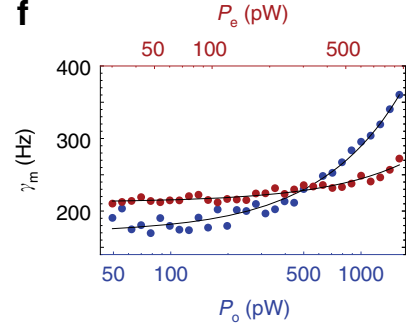

Fig. 2 Coherent scattering parameter measurements. a The reflection $\left|S_{\mathrm{jj}}\right|^{2}(j=e, 0)$ and bidirectional transduction $\zeta:=\left|S_{\mathrm{eo}}\right|\left|S_{\mathrm{oe}}\right|$ parameters as a function of signal tone detuning $\delta_{\mathrm{j}}=\omega-\left(\omega_{\mathrm{d}, \mathrm{j}}+\omega_{\mathrm{m}}\right)$ for fixed pump powers $P_{\mathrm{e}}=601 \mathrm{pW}$ and $P_{\mathrm{o}}=625 \mathrm{pW}$. The dots represent the experimental data while the solid lines show the theoretical prediction with $\gamma_{\mathrm{m}}$ as the only fit parameter. $\mathbf{b}$ Measured photon number transduction efficiency as a function of microwave and optical pump powers. c Measured transduction efficiency with respect to optical pump power for fixed $P_{\mathrm{e}}=601 \mathrm{pW}$. d Measured transduction efficiency with respect to microwave pump power for fixed $P_{\mathrm{o}}=625 \mathrm{pW}$. In (c) and (d), the error bars are the standard deviation of three independent measurement runs and solid lines are theory with interpolated $\gamma_{m}$ (from (f)) and no other free parameters. e The coupling efficiency of the microwave resonator to the waveguide $\eta_{\mathrm{e}}=\kappa_{\mathrm{ex}, \mathrm{e}} / \kappa_{\mathrm{e}}$, extracted from broadband reflection measurements, as a function of optical (blue, $P_{\mathrm{e}}=601 \mathrm{pW}$ ) and microwave (red, $P_{\mathrm{o}}=625 \mathrm{pW}$ ) pump tones. $\mathbf{f}$ Intrinsic mechanical decoherence rate $\gamma_{\mathrm{m}}$ versus optical (blue, $P_{\mathrm{e}}=601 \mathrm{pW}$ ) and microwave (red, $P_{\mathrm{o}}=625 \mathrm{pW}$ ) pump powers extracted from (c) and (d) with Eq. (3) and $\gamma_{m}$ as only fit parameter. Solid lines in (f) are linear fits to the data.

Figure $2 \mathrm{~b}$ shows the total transduction efficiency for different pump power combinations with microwave and optical pump powers ranging from 30 to $953 \mathrm{pW}$ and 48 to $1561 \mathrm{pW}$, respectively. Figure $2 \mathrm{c}$, d shows the efficiency versus $P_{\mathrm{o}}\left(P_{\mathrm{e}}\right)$ for fixed microwave (optical) pump power $P_{\mathrm{e}}=601\left(P_{\mathrm{o}}=625\right) \mathrm{pW}$. As expected, the transduction efficiency rises with increasing pump powers and reaches a maximum of $\zeta=1.2 \%$. The internal transduction efficiency is significantly higher $\left(\zeta /\left(\eta_{\mathrm{o}} \eta_{\mathrm{e}}\right) \leq 135 \%\right)$ because both the microwave resonator as well as the optical cavity are highly undercoupled with coupling ratios of $\eta_{\mathrm{o}}=0.11$ and $\eta_{\mathrm{e}}$ ranging between 0.07 and 0.18 when both pumps are on. The increase in the intrinsic loss rate of microwave $\kappa_{\text {in,e }}$ and mechanical resonator $\gamma_{\mathrm{m}}$ at higher pump powers are shown in Fig. $2 \mathrm{e}$ and $\mathrm{f}$ caused by considerable heating related to (especially optical) photon absorption. This results in the degradation of the microwave and mechanical quality factors and consequently reduces the waveguide coupling efficiency, the cooperativities and the total transduction efficiency (see Supplementary Note 5).

Sideband resolution and amplification. In the nonsideband resolved limit the contribution of the counter-rotating term of the Hamiltonian $\hat{H}_{\mathrm{CR}, \mathrm{o}}$ is nonnegligible, resulting in a transduction process that cannot be fully noise- free. This interesting effect can be correctly described by introducing an amplification of the signal tone with (in the absence of thermal noise) quantum limited gain $\mathcal{G}_{0}$ (see Supplementary Note 1). In contrast, the microwave resonator is in the resolved-sideband condition $4 \omega_{\mathrm{m}}>$ $\kappa_{\mathrm{e}}$, so that the signal tone amplification due to electromechanical interaction is negligible $\mathcal{G}_{\mathrm{e}} \simeq 1$. This results in the total, power independent, bidirectional conversion gain of $\mathcal{G}=\mathcal{G}_{\mathrm{e}} \mathcal{G}_{\mathrm{o}} \simeq \mathcal{G}_{\mathrm{o}}$, which turns out to be directly related to the minimum reachable phonon occupation:

$$
\langle n\rangle_{\min }=\frac{\left(\Delta_{\mathrm{o}}-\omega_{\mathrm{m}}\right)^{2}+\kappa_{\mathrm{o}}^{2} / 4}{4 \Delta_{\mathrm{o}} \omega_{\mathrm{m}}}=\mathcal{G}_{\mathrm{o}}-1,
$$

induced by optomechanical quantum backaction when the mechanical resonator is decoupled from its thermal bath ${ }^{32}$. Due to this amplification process the measured transduction efficiency in Fig. 2a is about 110 times larger than one would expect from a model that does not include gain effects for the chosen detuning, and adds the equivalent of at least one half of a vacuum noise photon to the input of the transducer in our case of heterodyne detection (for $\eta_{j}=1$ and $\mathcal{G} \gg 1$ ). However, it turns out that this noise limitation, which might in principle be overcome with efficient feedforward ${ }^{9}$, sideband suppression ${ }^{33,34}$, or sideband resolution $^{35}$, accounts for only about $0.1 \%$ of the total conversion noise observed in our system. The total transduction (including gain) can be written in terms of the susceptibilities of the electromagnetic modes $\chi_{\mathrm{j}}^{-1}(\omega)=i\left(\Delta_{\mathrm{j}}-\omega\right)+\kappa_{\mathrm{j}} / 2$ and the mechanical resonator $\chi_{\mathrm{m}}^{-1}(\omega)=i\left(\omega_{\mathrm{m}}-\omega\right)+\gamma_{\mathrm{m}} / 2$ as:

$$
\zeta=\left|\frac{\sqrt{\kappa_{\mathrm{ex}, \mathrm{e}} \kappa_{\mathrm{ex}, \mathrm{o}}} G_{\mathrm{e}} G_{\mathrm{o}} \chi_{\mathrm{e}} \chi_{\mathrm{o}}\left[-\chi_{\mathrm{m}}+\tilde{\chi}_{\mathrm{m}}\right]}{1+\left[\chi_{\mathrm{m}}-\tilde{\chi}_{\mathrm{m}}\right]\left[G_{\mathrm{e}}^{2}\left(\chi_{\mathrm{e}}-\tilde{\chi}_{\mathrm{e}}\right)+G_{\mathrm{o}}^{2}\left(\chi_{\mathrm{o}}-\tilde{\chi}_{\mathrm{o}}\right)\right]}\right|^{2},
$$

where $\tilde{\chi}_{\mathrm{j}}(\omega)=\chi_{\mathrm{j}}(-\omega)^{*}$.

Equation (3) can be decomposed into a product of the conversion gain $\mathcal{G}$ and the pure conversion efficiency $\theta$, i.e., $\zeta:=\mathcal{G} \times \theta$, for frequencies in the vicinity of $\omega_{\mathrm{m}}$ (see Supplementary Note 1). Equation (2) shows that the signal amplification depends only on the resonator linewidth and the detuning and is not directly related to the $\propto \hat{a}^{\dagger} \hat{b}^{\dagger}$ interaction term or the pump power $^{31}$. This can be understood by the alternative interpretation that the gain represents the ratio of the transduced upper sideband to the difference between upper and lower sideband at each cavity. Therefore, it is instructive to measure the transducer parameters as a function of optical pump detuning as shown in Fig. 3a. While changing the optical detuning, we also vary the pump power in order to keep the optical intracavity photon number constant at $n_{\mathrm{d}, \mathrm{o}}=0.185 \pm 0.015$. This way it is possible to investigate the influence of $\Delta_{\mathrm{o}}$ at a constant optomechanical 

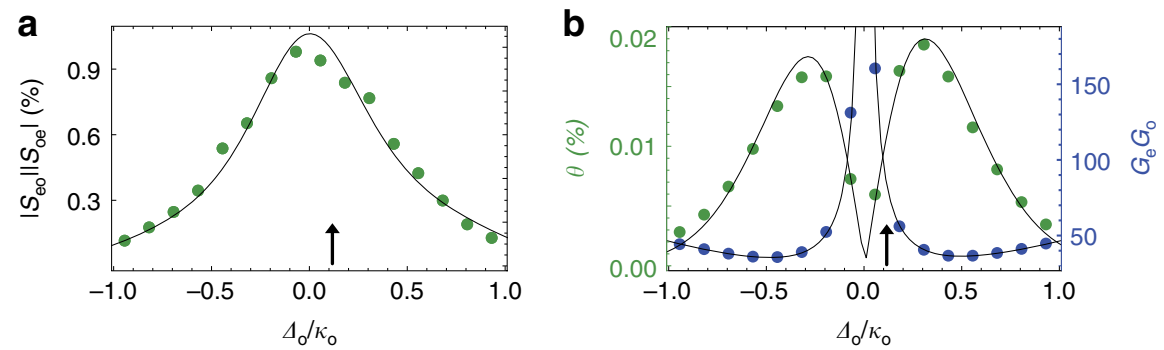

Fig. 3 Pure and amplified conversion. a Measured total transduction efficiency $\zeta:=\left|S_{\text {eo }}\right|\left|S_{\text {oel }}\right|$ with respect to pump detuning $\Delta_{\circ}$ of the nonsideband resolved optical cavity for a constant intracavity photon number $n_{\mathrm{d}, \mathrm{o}}=0.185$. $\mathbf{b}$ Pure and-in the absence of thermal noise-noiseless conversion $\theta$ (green dots) as well as the total conversion gain $\mathcal{G}$ (blue dots) that gives rise to amplified vacuum noise. $\theta$ is extracted from (a) by dividing the measured total transduction by the calculated gain using Supplementary Eqs. (16) and (17). While $\mathcal{G}_{0}$ diverges for $\Delta_{0} \rightarrow 0$, the optomechanical damping rate drops to zero and leads to a vanishing pure conversion $\theta$. The arrows in both panels indicate the detuning used in Figs. 2 and 4.

coupling $G_{\mathrm{o}}=g_{0, \mathrm{o}} \sqrt{n_{\mathrm{d}, \mathrm{o}}}$. The measured total transduction efficiency is shown in Fig. 3a and reaches $\zeta \approx 1 \%$ at $\Delta_{\mathrm{o}} \approx 0$ for the chosen pump powers in agreement with Fig. $2 c$, d. We can now separate the measured transduction (Eq. (3)) into conversion gain and pure conversion, as shown in Fig. $3 \mathrm{~b}$. The gain shows the expected steep increase at $\Delta_{\mathrm{o}} \rightarrow 0$ where the pure conversion $\theta$ approaches zero for equal cooling and amplification rates. Around $\Delta_{\mathrm{o}}=\kappa_{\mathrm{o}} / 2$ on the other hand, where $\langle n\rangle_{\min }$ reaches its minimum of roughly $\kappa_{\mathrm{o}} / 4 \omega_{\mathrm{m}} \approx 30$, also the gain reaches its minimum and the noiseless part (at zero temperature) of the total (internal) conversion process shows its highest efficiency of $\theta=$ $0.019 \%\left(\theta /\left(\eta_{\mathrm{e}} \eta_{\mathrm{o}}\right)=1.6 \%\right)$.

Added noise. Another important figure of merit, not only for quantum applications, is the amount of added noise quanta ${ }^{36}$, usually an effective number referenced to the input of the device. For clarity with regards to the physical origin and the actual measurement of the noise power, in the following we define the total amount of added noise quanta $n_{\text {add, } j}$ added to the input signal $S_{\mathrm{in}, \mathrm{j}}$ after the transduction process as $S_{\text {out }, \mathrm{j}}=\zeta S_{\mathrm{in}, \mathrm{j}}+n_{\mathrm{add}, \mathrm{j}}$. Figure $4 \mathrm{a}, \mathrm{b}$ shows the measured conversion noise $n_{\mathrm{add}, \mathrm{j}}$ as a function of frequency $\delta_{\mathrm{j}}$ for the same powers and detunings as in Fig. 2a. At these powers our device adds $n_{\text {add,o(e) }}=224(145)$ noise quanta to the output of the microwave resonator (optical cavity), corresponding to an effective input noise of $n_{\text {add, } j} / \zeta$. The noise floor originates from the calibrated measurement system and in case of the microwave port to a small part also from an additional broadband resonator noise, cf. Fig. $4 \mathrm{~b}$. The solid lines are fits to the theory with the mechanical bath occupation $\bar{n}_{\mathrm{m}}$ as the only fit parameter (see Supplementary Note 4).

The fitted effective mechanical bath temperature as a function of pump powers is shown in Fig. 4c. It reveals the strong optical pump dependent mechanical mode heating (blue), while the microwave pump (red) has a negligible influence on the mechanical bath. Fig. $4 \mathrm{~d}$ shows the measured total added noise at the output of the microwave resonator and optical cavity as a function of optical pump power. The noise added to the optical output (blue) increases with pump power due to absorption heating and increasing optomechanical coupling rate $G_{0}$, while the degradation of the resonator-waveguide coupling efficiency $\eta_{e}$ explains the decreasing $n_{\text {add,e }}$ at higher optical powers for the microwave output noise (red), see Fig. 2e. The intersection of the two noise curves occurs at $\mathcal{C}_{\mathrm{e}} \simeq \mathcal{C}_{\mathrm{o}}$ with cooperativities $C_{\mathrm{j}}$ as defined above, and shows that the optical and microwave resonators share the same mechanical thermal bath. The power dependence is in full agreement with theory (solid lines) and demonstrates that the thermal mechanical population is the dominating origin of the added transducer noise.
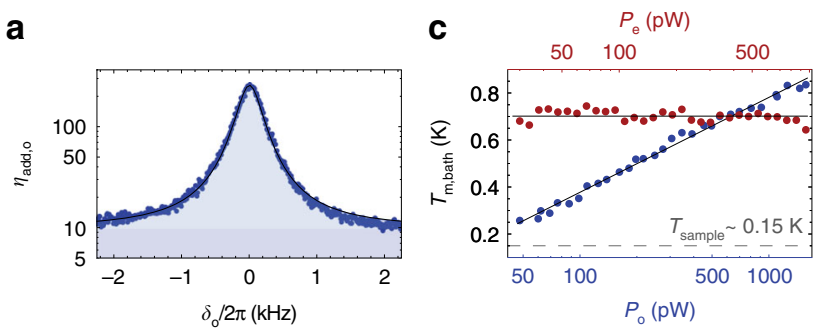

b

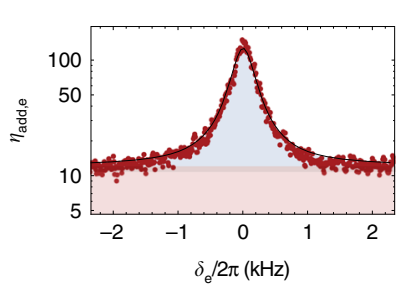

d

$C_{\mathrm{o}} / C_{\mathrm{e}}$

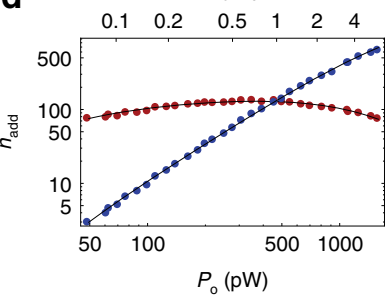

Fig. 4 Conversion noise properties. Measured noise spectra at the device output for the optical cavity (a) and the microwave resonator (b) as a function of the signal tone detuning $\delta_{\mathrm{j}}=\omega-\left(\omega_{\mathrm{d}, \mathrm{j}}+\omega_{\mathrm{m}}\right)$ at fixed pump powers $P_{\mathrm{e}}=601 \mathrm{pW}$ and $P_{\mathrm{o}}=625 \mathrm{pW}$ in units of added noise quanta. a The dark blue region represents the two-quadrature noise added by the optical measurement chain; the light blue region indicates the thermal mechanical noise added to the converted optical output signal. b Bottom light red region represents the two-quadrature background noise from the microwave measurement chain. The central dark red region indicates a small amount of broadband resonator noise and the light blue region the transduction noise due to the thermal population of the mechanical mode. In both panels, fits to one common mechanical bath $n_{m}$ are shown in black. c Mechanical bath temperature $T_{\text {m,bath }}$ extracted as only fit parameter from fits to the measured output noise as in (a) and (b) with respect to optical (blue dots, $P_{\mathrm{e}}=601 \mathrm{pW}$ ) and microwave (red dots, $P_{\mathrm{o}}=625 \pm 19 \mathrm{pW}$ ) pump power. Black lines show fits to the data with the logarithmic growth function $T_{\mathrm{m} \text {,bath }}=0.18 \log _{\mathrm{e}}\left(P_{\mathrm{o}}\right)-0.47$ and $T_{\mathrm{m} \text {, bath }}=0.70 \mathrm{~K}$ respectively. The dashed line indicates the thermalized mechanical mode temperature $T_{\text {sample }}$ when both pumps are off. $\mathbf{d}$ Microwave (red, $n_{\text {add,e }}$ ) and optical (blue, $n_{\text {add,o }}$ ) added noise photons at the output with respect to optical pump power $\left(P_{\mathrm{e}}=601\right.$ $\mathrm{pW})$. The full theory based on an interpolation of $T_{\mathrm{m}, \text { bath }}$ from (c) is shown as black lines.

\section{Discussion}

In conclusion, we demonstrated an efficient bidirectional and chip-scale microwave-to-optics transducer using pump powers orders of magnitude lower than comparable all-integrated ${ }^{11,13,15}$ approaches. Low pump powers are desired to limit the heat load of the cryostat and to minimize on-chip heating, which is 
particularly important for integrated devices because of their limited heat dissipation at millikelvin temperatures. Due to the standard material choice involving only silicon and aluminum, our device can be easily integrated with other elements of superconducting circuits as well as silicon photonic and phononic devices in the future.

The two main challenges ahead are the reduced pure conversion efficiency and the optical heating that adds incoherent noise to the converted signal. We expect that both can be solved with design improvements in combination with new measurement techniques. Specifically, starting from the observed pure efficiency of $0.019 \%$ a factor of up to nearly two orders of magnitude could be gained with better waveguide coupling geometries in combination with fabrication optimization, e.g., by using surface cleaning and the reduction of humidity ${ }^{37}$. Improving the sideband resolution by increasing the mechanical frequency ${ }^{35}$ could yield another factor of up to 25 assuming the same cooperativities can be achieved. Going to the high cooperativity limit would then yield the remaining fraction needed for unity total conversion efficiency. This will certainly require a very effective mitigation of the optical pump power dependent mechanical heating and the associated linewidth degradation that is also required for noisefree conversion. Nevertheless, with better chip thermalization, reduced optical absorption and low duty cycle pulsed measurements this should be feasible. Moreover, it has already been shown that pulsed pump-probe type experiments together with high efficiency heralding measurements can be used for postselecting rare successful conversion or entanglement generation events for low-noise low-efficiency devices ${ }^{12,38}$.

In terms of near-term classical receiver and modulation applications, an important figure of merit is the voltage required to induce an optical phase shift of $\pi$. We are able to reach a value as low as $V_{\pi}=16 \mu \mathrm{V}$ (see Supplementary Note 6), comparable with typical zero point fluctuations in superconducting circuits, nearly a factor 9 lower than the previously reported record ${ }^{19}$, and almost $10^{12}$ times more power efficient than commercial passive and wide-band unidirectional electro-optic modulators at $\mathrm{X}$ band gigahertz frequencies.

\section{Data availability}

The data and code used to produce the results of this paper are available at https://doi. org/10.5281/zenodo. 3961562 .

Received: 16 July 2020; Accepted: 6 August 2020;

Published online: 08 September 2020

\section{References}

1. Kimble, H. J. The quantum internet. Nature 453, 1023-1030 (2008).

2. Blais, A., Huang, R.-S., Wallraff, A., Girvin, S. M. \& Schoelkopf, R. J. Cavity quantum electrodynamics for superconducting electrical circuits: an architecture for quantum computation. Phys. Rev. A 69, 062320 (2004)

3. Awschalom, D. D., Hanson, R., Wrachtrup, J. \& Zhou, B. B. Quantum technologies with optically interfaced solid-state spins. Nat. Photon. 12, 516-527 (2018).

4. Burkard, G., Gullans, M. J., Mi, X. \& Petta, J. R. Superconductorsemiconductor hybrid-circuit quantum electrodynamics. Nat. Rev. Phys. 2, 129-140 (2020).

5. Kurpiers, P., Walter, T., Magnard, P., Salathe, Y. \& Wallraff, A. Characterizing the attenuation of coaxial and rectangular microwave-frequency waveguides at cryogenic temperatures. EPJ Quantum Technol. 4, 8 (2017).

6. Lambert, N. J., Rueda, A., Sedlmeir, F. \& Schwefel, H. G. L. Quantum information technologies: coherent conversion between microwave and optical photons-an overview of physical implementations. Adv. Quantum Technol. 3, 2070011 (2020).

7. Lauk, N. et al. Perspectives on quantum transduction. Quantum Sci. Technol. 5, 020501 (2020).
8. Stannigel, K., Rabl, P., Sørensen, A. S., Zoller, P. \& Lukin, M. D. Optomechanical transducers for long-distance quantum communication. Phys. Rev. Lett. 105, 220501 (2010).

9. Higginbotham, A. P. et al. Harnessing electro-optic correlations in an efficient mechanical converter. Nat. Phys. 14, 1038-1042 (2018).

10. Vainsencher, A., Satzinger, K. J., Peairs, G. A. \& Cleland, A. N. Bi-directional conversion between microwave and optical frequencies in a piezoelectric optomechanical device. Appl. Phys. Lett. 109, 033107 (2016).

11. Jiang, W. et al. Efficient bidirectional piezo-optomechanical transduction between microwave and optical frequency. Nat. Commun. 11, 1166 (2020).

12. Forsch, M. et al. Microwave-to-optics conversion using a mechanical oscillator in its quantum ground state. Nat. Phys. 16, 69-74 (2020).

13. Han, X. et al. Cavity piezo-mechanics for superconducting-nanophotonic quantum interface. Nat. Commun. 11, 3237 (2020).

14. Rueda, A. et al. Efficient microwave to optical photon conversion: an electrooptical realization. Optica 3, 597-604 (2016).

15. Fan, L. et al. Superconducting cavity electro-optics: a platform for coherent photon conversion between superconducting and photonic circuits. Sci. Adv. 4, eaar4994 (2018).

16. Hease, W. et al. Cavity quantum electro-optics: microwave-telecom conversion in the quantum ground state. arXiv (2020). https://arxiv.org/abs/2005.12763v1.

17. Safavi-Naeini, A. H., Van Thourhout, D., Baets, R. \& Van Laer, R. Controlling phonons and photons at the wavelength scale: integrated photonics meets integrated phononics. Optica 6, 213-232 (2019).

18. Keller, A. J. et al. Al transmon qubits on silicon-on-insulator for quantum device integration. Appl. Phys. Lett. 111, 042603 (2017)

19. Bagci, T. et al. Optical detection of radio waves through a nanomechanical transducer. Nature 507, 81-85 (2014).

20. Simonsen, A. et al. Magnetic resonance imaging with optical preamplification and detection. Sci. Rep. 9, 18173 (2019).

21. Regal, C. A. \& Lehnert, K. W. From cavity electromechanics to cavity optomechanics. J. Phys.: Conf. Ser. 264, 012025 (2011).

22. Safavi-Naeini, A. H. \& Painter, O. Proposal for an optomechanical traveling wave phonon-photon translator. New J. Phys. 13, 013017 (2011).

23. Barzanjeh, S., Abdi, M., Milburn, G. J., Tombesi, P. \& Vitali, D. Reversible optical-to-microwave quantum interface. Phys. Rev. Lett. 109, 130503 (2012).

24. Hill, J. T., Safavi-Naeini, A. H., Chan, J. \& Painter, O. Coherent optical wavelength conversion via cavity optomechanics. Nat. Commun. 3, 1196 (2012).

25. Lecocq, F., Clark, J. B., Simmonds, R. W., Aumentado, J. \& Teufel, J. D. Mechanically mediated microwave frequency conversion in the quantum regime. Phys. Rev. Lett. 116, 043601 (2016).

26. Fink, J. M., Kalaee, M., Norte, R., Pitanti, A. \& Painter, O. Efficient microwave frequency conversion mediated by a photonics compatible silicon nitride nanobeam oscillator. Quantum Sci. Technol. 5, 034011 (2020).

27. Safavi-Naeini, A. H. et al. Squeezed light from a silicon micromechanical resonator. Nature 500, 185-189 (2013).

28. Barzanjeh, S. et al. Stationary entangled radiation from micromechanical motion. Nature 570, 480-483 (2019).

29. Peruzzo, M., Trioni, A., Hassani, F., Zemlicka, M. \& Fink, J. M. Surpassing the resistance quantum with a geometric superinductor. arXiv (2020). https:// arxiv.org/abs/2007.01644.

30. Dieterle, P. B., Kalaee, M., Fink, J. M. \& Painter, O. Superconducting cavity electromechanics on a silicon-on-insulator platform. Phys. Rev. Appl. 6, 014013 (2016).

31. Andrews, R. W. et al. Bidirectional and efficient conversion between microwave and optical light. Nat. Phys. 10, 321-326 (2014).

32. Aspelmeyer, M., Kippenberg, T. J. \& Marquardt, F. Cavity optomechanics. Rev. Mod. Phys. 86, 1391-1452 (2014).

33. Asjad, M., Zippilli, S. \& Vitali, D. Suppression of stokes scattering and improved optomechanical cooling with squeezed light. Phys. Rev. A 94, 051801 (2016).

34. Lau, H.-K. \& Clerk, A. A. Ground-state cooling and high-fidelity quantum transduction via parametrically driven bad-cavity optomechanics. Phys. Rev. Lett. 124, 103602 (2020).

35. Kalaee, M. et al. Quantum electromechanics of a hypersonic crystal. Nat. Nanotechnol. 14, 334-339 (2019).

36. Zeuthen, E., Schliesser, A., Sørensen, A. S. \& Taylor, J. M. Figures of merit for quantum transducers. Quantum Sci. Technol. 5, 034009 (2020).

37. Sekoguchi, H., Takahashi, Y., Asano, T. \& Noda, S. Photonic crystal nanocavity with a Q-factor of 9 million. Opt. Express 22, 916-924 (2014).

38. Zhong, C. et al. Proposal for heralded generation and detection of entangled microwave-optical-photon pairs. Phys. Rev. Lett. 124, 010511 (2020).

\section{Acknowledgements}

We thank Yuan Chen for performing supplementary FEM simulations and Andrew Higginbotham, Ralf Riedinger, Sungkun Hong, and Lorenzo Magrini for valuable discussions. This work was supported by IST Austria, the IST nanofabrication facility (NFF), the European Union's Horizon 2020 research and innovation program under 
grant agreement no. 732894 (FET Proactive HOT) and the European Research Council under grant agreement no. 758053 (ERC StG QUNNECT). G.A. is the recipient of a DOC fellowship of the Austrian Academy of Sciences at IST Austria. W.H. is the recipient of an ISTplus postdoctoral fellowship with funding from the European Union's Horizon 2020 research and innovation program under the Marie Sklodowska-Curie grant agreement no. 754411. J.M.F. acknowledges support from the Austrian Science Fund (FWF) through BeyondC (F71), a NOMIS foundation research grant, and the EU's Horizon 2020 research and innovation program under grant agreement no. 862644 (FET Open QUARTET).

\section{Author contributions}

G.A., M.W., and S.B. performed and analyzed the measurements. S.B. and G.A. contributed to the theoretical model. G.A. designed the transducer device. M.W., G.A., A.R., and W.H. built the experimental setup. M.W., E.R., and G.A. contributed to sample fabrication. F.H. tapered optical fibers used for optomechanical characterization tests. G.A., M.W., S.B., and J.M.F. wrote the paper. J.M.F. supervised the research.

\section{Competing interests}

The authors declare no competing interests.

\section{Additional information}

Supplementary information is available for this paper at https://doi.org/10.1038/s41467020-18269-z.
Correspondence and requests for materials should be addressed to J.M.F.

Peer review information Nature Communications thanks the anonymous reviewers for their contribution to the peer review of this work. Peer reviewer reports are available.

Reprints and permission information is available at http://www.nature.com/reprints

Publisher's note Springer Nature remains neutral with regard to jurisdictional claims in published maps and institutional affiliations.

(c) (i) Open Access This article is licensed under a Creative Commons Attribution 4.0 International License, which permits use, sharing, adaptation, distribution and reproduction in any medium or format, as long as you give appropriate credit to the original author(s) and the source, provide a link to the Creative Commons license, and indicate if changes were made. The images or other third party material in this article are included in the article's Creative Commons license, unless indicated otherwise in a credit line to the material. If material is not included in the article's Creative Commons license and your intended use is not permitted by statutory regulation or exceeds the permitted use, you will need to obtain permission directly from the copyright holder. To view a copy of this license, visit http://creativecommons.org/ licenses/by/4.0/.

(C) The Author(s) 2020 